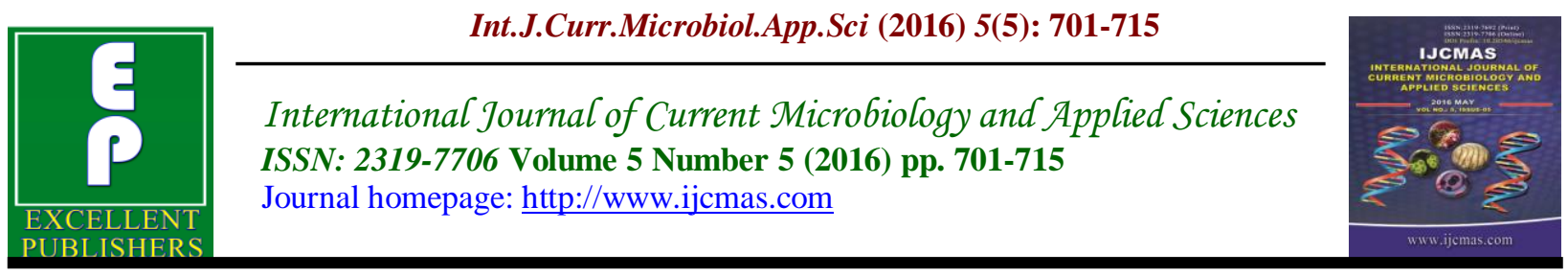

Original Research Article

http://dx.doi.org/10.20546/ijcmas.2016.505.071

\title{
Non Invasive Diagnosis of Esophageal Varices: Can it Replace Screening Endoscopy?
}

\author{
Elshazly Abd Elhady Sheta ${ }^{1}$, Mohamed Yousef ${ }^{1}$, Sherief Abd-Elsalam ${ }^{1 *}$, \\ Rania Essam Eldeen Mohamed ${ }^{2}$, Ayat Ismail ${ }^{1}$, Ferial El-Kalla ${ }^{1}$, Loai Mansour ${ }^{1}$, \\ Abdelrahman Kobtan', Mohamed Elhendawy ${ }^{1}$ and Hala M. Elsabagh ${ }^{4}$ \\ ${ }^{1}$ Tropical Medicine \& Infectious Diseases Department, Tanta University, Egypt \\ ${ }^{2}$ Radiology Department, Tanta University, Egypt \\ ${ }^{3}$ Tropical Medicine \& Infectious Diseases Department, Kafr Elsheikh University, Egypt \\ ${ }^{4}$ Public health and community medicine Department, Tanta University \\ Faculty of Medicine, Tanta, Egypt \\ *Corresponding author
}

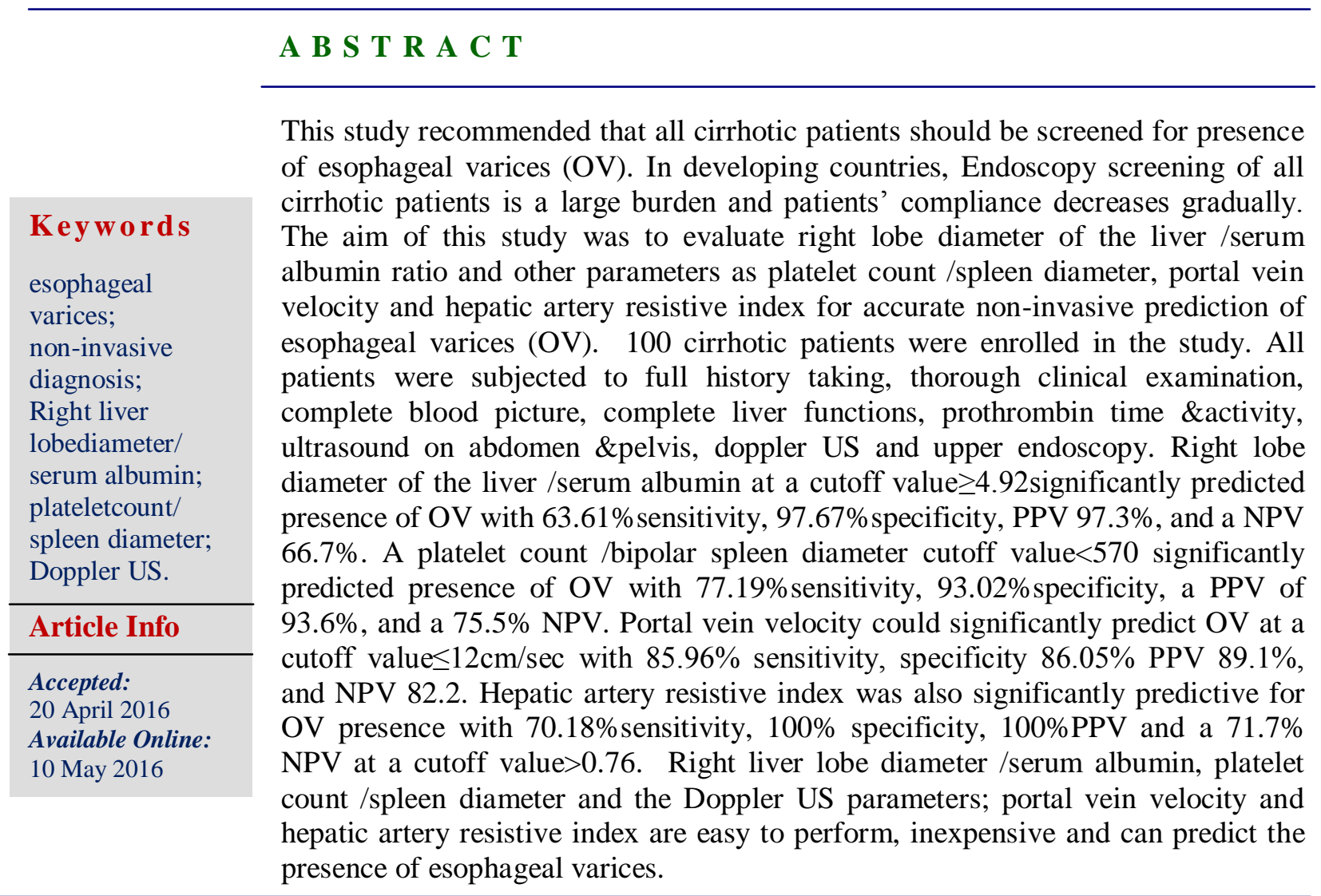

\section{Introduction}

Esophagealvarices (EV) development is one of the major complications of liver
Cirrhosis (Tsochatziset al., 2014), with an estimated prevalence of approximately $50 \%$ 
in compensated cirrhosis and 80\% with decompensated patients (Arguedas et al., 2002).

The mortality from each episode of variceal bleeding ranges from $17-57 \%$. Bleeding episodes can be predicted by the presence of "red cherry spots " on the varices, and by variceal size (Jensen, 2002).

Varices initially develop in 5\%-10\% of cirrhotic patients every year and the rate of transition from small to large varices ranges between 5\% and 30\% per year (Garcia-Tsao et al., 2007). Large esophagealvarices are at a greater risk of bleeding, possibly due to a higher variceal wall tension (Navens et al., 1998).

Prophylactic endoscopic variceal ligation can decrease the incidence of a first variceal bleeding and mortality in patients with liver cirrhosis who have large varices (Madhotra et al., 2002). All guidelines stress on screening endoscopy for early detection of EV in cirrhotic patients with portal hypertension.

On the other hand, it was found that many subjects screened either do not have varices or have non-risky varices not requiring prophylactic therapy. Endoscopic screening in these patients is a burden to endoscopic units. In addition, patient compliance with the screening program may be reduced over time (De Franchis, 2008).

For these reasons, several studies have examined how to identify patients with varices using non-invasive or minimally invasive methods to avoid endoscopy in patients with a low risk of varices. These studies include biochemical, clinical and ultrasound parameters.

So, the aim of this study was to evaluate right lobe diameter of the liver /serum albumin ratio and other parameters as platelet count /spleen diameter, portal vein velocity and hepatic artery resistive index for prediction of varices.

Portal hypertension $(\mathrm{PH})$, a progressive complication of liver cirrhosis, is defined as a pathological increase in the portal venous pressure between the portal vein and the inferior vena cava to higher than the normal (Normal range is $\leq 5 \mathrm{mmHg}$ ) (De Franchis et al., 2014).

Clinically significant PH (Hepatic venous pressure gradient $\geq 10 \mathrm{mmHg}$ ) is necessary for the development of esophageal varices $(\mathrm{EV})$ and variceal bleeding along with the development of decompensation (Ripoll et al., 2013).

Variceal bleeding occurs in $25-40 \%$ of patients with cirrhosis. Each episode of variceal bleeding is associated with approximately $20 \%$ mortality rate ${ }^{\mathbf{1 6}}$. One in four patients with EV will likely suffer an episode of variceal bleeding over a period of two years (Chawla et al., 2012).

A recent database analysis by D'Amico et al., (2014)18 showed that the cumulative incidence of varices at 10 and 20 years was $44 \%$ and $53 \%$, respectively, suggesting an over- estimation in previous studies. Numerous lines of evidence suggest that varices develop and enlarge with time.

Screening of EV is recommended for all patients at the time of initial diagnosis of cirrhosis. On screening for EV, 9-36\% of patients with cirrhosis are found to have esophageal varices.

There is universal acceptance that endoscopy is the 'gold standard' for diagnosing gastro-esophageal varices. However, a generalized screening program of periodical upper endoscopy in cirrhotic 
patients is costly and associated with low patient compliance, as the procedure is invasive and may be poorly accepted by the patients if repeatedly required. Moreover, sedation of cirrhotic patients to perform upper endoscopy may be hazardous and diagnostic upper endoscopy may contribute to bacterial infections due to disruption of the normal barriers. For these reasons, the selection of patients who may be at risk of having $\mathrm{OV}$, especially those at risk of rupture would be highly beneficial and cost effective.

\section{Materials and Methods}

This prospective study included cirrhotic patients with liver cirrhosis under investigation and /or treatment at outpatient clinics, patients admitted to Tropical medicine department of Tanta university hospitals or patients referred to the hospital endoscopy unit for endoscopic screening for the presence of esophageal varices between January and April, 2015.

Our study was approved by the ethical committee of faculty of medicine, Tanta University. The research team recruited potential participants, and explained to each patient the aim of the research, and a written consent was obtained from all participants in the study.

The study included patients with HCV or HBV related cirrhosis. Patients with previous or active gastrointestinal bleeding, patients who had previously undergone injection sclerotherapy and/ or band ligation for esophageal varices, patients who were receiving beta blockers, patients with hepatocellular carcinoma, patients with primary haemorrhagic disorders were excluded from the study.

All patients were subjected to full history taking and through clinical examination. Patients were defined clinically according to the modified Child-Turcotte-Pugh classification into Child class $\mathrm{A}, \mathrm{B}$ or $\mathrm{C}$. Laboratory investigations were done for all patients including $\mathrm{HCV}$ and $\mathrm{HBV}$ viral markers, schistosomal antibodies, complete liver function tests, prothrombin time and activity and complete blood picture.

Upper endoscopy was performed for all patients enrolled in the study. Patients were requested to fast overnight and received premedication in the form of xylocaine local spray above the tongue and nasopharynx. Midazolam 3-5mg I.V was also given for most patients before the procedure. Endoscopy was performed using Pentax EG2985. The patient lay on the examination trolley/stretcher on the left side with the intravenous access line preferably in the right arm. The endoscope was inserted under direct vision. Examination of the entire oesophagus, stomach, and proximal duodenum, wherever possible was done.

Abdominal ultrasonography was done for all patients enrolled in the study at the Tropical Medicine Department using a Toshiba 770 $25 \mathrm{~A} \circledR$ with convex probe, $3.5 \mathrm{MH}$. Patients were examined after overnight fasting in the supine position. Aquatic gel was spread as a film on the abdomen of the patient to prevent interposition of air between the transducer and the skin. Screening was done through several longitudinal, oblique and transverse cuts. Measurements were taken in quiet respiration. Ultrasonography evaluation included the appearance of the liver as regards size (average, enlarged or shrunken), echo pattern of the liver (normal, bright, coarse or heterogeneous), established cirrhosis signs (shrunken liver, uneven hepatic margins, increased parenchymatous reflectivity, coarseness, increased echographic contrast between right lobe of liver and right kidney, hypertrophied caudate lobe and attenuated hepatic 
veins).Right lobe diameter of the liver in midclavicular line was measured. So, the presence of hepatic focal lesions, portal vein (PV) patency and diameter as well as presence of ascites (mild, moderate or marked)were assessed. Splenic size was measured in a supine or lateral recumbent position. As the length of the spleen is normally less than $130 \mathrm{~mm}$, enlargement was classified as mild (130-160mm), moderate $(160-200 \mathrm{~mm})$ and huge (> 200 $\mathrm{mm})$ splenomegaly, (Eichner, 1979). Calculation of platelet count/spleen diameter ratio $(\mathrm{PC} / \mathrm{SD})$ was done.

Finally, Duplex-Doppler Ultrasound was performed for all patients. Patients were examined after fasting overnight. Doppler sonographic examinations were performed at the Radiology Department with color Doppler ultrasound scanner using a Siemens G60® Ultrasound System with convex probe 3.5 MHZ for identification of portal vein, hepatic artery (HA), splenic artery and splenic vein. Measurements were performed with the subjects in the supine position, with breath-holding after shallow inspiration with an angle less than $60^{\circ}$ between the vessel and the ultrasound beam. Measurement of portal venous hemodynamics for each parameter was taken as the mean of three measurements. The PV was visualized in Bmode, and the sample volume cursor was shifted to the middle portal trunk at the level of the crossing point of the HA. The computer software of the US machine calculated the maximum portal flow velocity during suspended inspiration. Measurements of hepatic artery hemodynamics were taken. The mean of three successive cardiac cycles was obtained for each group of waveforms (Eichner et al., 1985). Hepatic artery resistive index was calculated by the computer software of the US machine.

\section{Statistical analysis}

The collected data were tabulated and statistically analyzed using SPSS (statistical package for social studies) version 19. Categorical variables were represented by number and percentage and observed differences were tested using Monte Carlo exact test. Numerical variables were presented as number and percentage. Mean differences were tested by student's $t$ test when comparing between two variables and analysis of variance $(\mathrm{F})$ when comparing more than two groups. When the $\mathrm{F}$ value was found significant, Bonferroni test was used to compare between each two means. Receiver operator characteristic (ROC) curve was used to determine the cutoff value for diagnosis, then the sensitivity, specificity, positive predictive value (PPV), negative predictive value (NPV) and accuracy of each cutoff variable were calculated. The level of significance was adopted at $\mathrm{p}$ value $<0.05$.

\section{Results and Discussion}

143 cirrhotic patients were invited to and assessed for participation in the study. 43 patients were excluded from the study (24 did not meet the inclusion and exclusion criteria and 19 refused to participate in the study). Finally, a total of 100 cirrhotic patients were enrolled in this study.

Regarding the cirrhotic patients enrolled in this study, their age ranged from $42-75$ years with a mean value of $54.030 \pm 7.223$. Hemoglobin ranged from 6.6-16 gm/dl with a mean value of $10.372 \pm 1.720$. White blood cells count ranged from $2-10.5 \times 103 / \mathrm{mm} 3$ with a mean value of $4.32 \pm 1.56 \times 103$. Platelets count ranged from 50-210 x109/L with mean value of $95.17 \pm 33.5 \times 109$.

Serum albumin in patients enrolled in this study ranged from 1.9-4 gm/dl with a mean value of $2.766 \pm 0.581$. Total serum bilirubin ranged from $0.5-9 \mathrm{mg} / \mathrm{dl}$ with a mean value 
of $2.109 \pm 1.391$. International normalized ratio (INR) ranged from 1-3.2 with a mean value of $1.499 \pm 0.388$. Liver right lobe diameter ranged from $9-16.5 \mathrm{~cm}$ with a mean of 12.219 \pm 1.590 . Right lobe (Rt. Lobe) / Albumin ratio ranged from 2.86-6.67 with a mean value of $4.553 \pm 0.818$. PV velocity ranged from $8-16.5 \mathrm{~cm} / \mathrm{sec}$. with mean velocity of $11.906 \pm 2.512$. Platelet count / spleen diameter ratio ranged from 301-750 with a mean value of $664 \pm 262$. Hepatic artery (HA) resistive index ranged from 0.60.9 with a mean value of $0.739 \pm 0.082$. Spleen diameter ranged from 110-240 mm with a mean value of $147.390 \pm 20.836$. Descriptive statistics of studied patients are shown in table (1).

Among 100 cirrhotic patients enrolled in the study, 43 patients had no varices. 10 patients were found to have grade I EV, 15 patients were found to have grade II EV and 32 were found to have grade III EV [table (2)].

Regarding right lobe diameter of the liver /serum albumin, there was a significant direct relation between right liver lobe diameter/serum albumin and EV grades. Patients with no varices had a mean ratio of $4.116 \pm 0.502$, whereas patients with grade I,II, and III, varices had a mean ratio value of $4.291 \pm 0.842,4.871 \pm 0.971$ and 5.073 \pm 0.742 respectively. Tukey's test showed a significant difference between absence of varices \& presence of grade II varices $(\mathrm{p}=$ 0.003 ) and between no\& grade III varices $(\mathrm{p}<.0 .001)$ and between grade II\&III $(\mathrm{P}=$ 0.014) [table (3)].Receiver Operator Characteristic (ROC) curve for of right lobe diameter of the liver /serum albumin as diagnostic parameter for the presence of $\mathrm{OV}$ is shown in figure (1).

As regards the platelet count/spleen diameter ratio (PC/SD), there was a significant inverse relation between $\mathrm{PC} / \mathrm{SD}$ and grades of EV. Patients with no varices had a mean PC/SD ratio of $866 \pm 197$, patients with grade I varices had a mean PC/SD ratio of $850 \pm 173$, patients with grade II varices had a mean PC/SD ratio of $473 \pm 56$ and patients with grade III varices had a mean value of $425 \pm 110$. There was statistically significant relation to the presence of EV (p.value $<0.001$ ). Tukey's test showed a significant difference between absence of varices \& grade II, III and between grade I \& II, III with a $\mathrm{p}$ value $<.0 .001$ [table (4)]. Receiver Operator Characteristic (ROC) curve for evaluation of Platelet count/bipolar spleen diameter as diagnostic parameter for the presence of $\mathrm{OV}$ is shown in figure (2).

As regards portal vein velocity, there was a significant inverse relation between portal vein velocity and grades of EV. Patients with no varices had portal vein velocity with a mean value of $14.021 \pm 1.651 \mathrm{~cm} / \mathrm{sec}$. The mean portal vein velocity in patients with

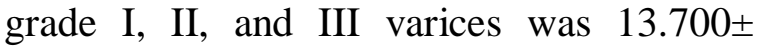

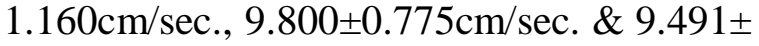
$0.617 \mathrm{~cm} / \mathrm{sec}$ respectively. There was a statistically significant relation to the presence of EV (p.value $<0.001)$. Tukey's test showed a significant difference between absence of varices \& grade II, III and between grade I\&II, III with p value <.0.001 [table (5)].Receiver Operator Characteristic (ROC) curve for evaluation of portal vein velocity as diagnostic parameter for the presence of OV is shown in figure (3).

Finally, regarding hepatic artery resistive index (HARI), there was a direct significant relation between hepatic artery resistive index and EV grades. Patients with no varices had a HARI with a mean value of $0.673 \pm 0.048$.Patients with grade I, II, and III varices had a mean HARI of $0.722 \pm$ $0.050, \quad 0.802 \pm 0.060$, and $0.805 \pm 0.058$ respectively. A statistically significant relation to the presence of $\mathrm{EV}$ 
(p.value<0.001) was observed. Tukey's test showed a significant difference between absence of varices \& grade I with $\mathrm{p}$ value 0.047 and between grade no \& grade II, III with $\mathrm{p}$ value $<.0 .001$ and between grade I\&II with value 0.002 and grade I\&III with $\mathrm{p}$ value $<0.001[$ table (6)]. Receiver Operator Characteristic (ROC) curve for evaluation of hepatic artery resistive index as diagnostic parameter for the presence of $\mathrm{OV}$ is shown in figure (4).

There was a significant correlation between esophageal varices grade and child classification. This correlation is shown in table (7). There was also significant correlation between right lobe diameter of liver/serum albumin \& platelet count /spleen diameter (PC/SD) as shown in figure (5).

Table.1 Descriptive Statistics of studied patients

\begin{tabular}{|c|c|c|c|c|c|c|}
\hline \multicolumn{7}{|c|}{ Descriptive Statistics } \\
\hline & & an & & Mean & \pm & SD \\
\hline Age (years) & 42 & - & 75 & 54.030 & \pm & 7.223 \\
\hline Hb (gm /dl) & 6.6 & - & 16 & 10.372 & \pm & 1.720 \\
\hline Platelet (cell/L) & 50.000 & - & 210.000 & 95.170 & \pm & 33.504 \\
\hline WBC $\left(\right.$ cell/mm $\left.\mathbf{m m}^{3}\right)$ & 2000 & - & 10500 & 4328.550 & \pm & 1561.152 \\
\hline Albumin (gm/dl) & 1.9 & - & 4 & 2.766 & \pm & 0.581 \\
\hline Bilirubin(mg /dl) & 0.5 & - & 9 & 2.109 & \pm & 1.391 \\
\hline INR & 1 & - & 3.2 & 1.499 & \pm & 0.388 \\
\hline Liver Right lobe diameter $(\mathrm{cm})$ & 9 & - & 16.5 & 12.219 & \pm & 1.590 \\
\hline Rt lobe / Albumin & 2.86 & - & 6.67 & 4.553 & \pm & 0.818 \\
\hline Platelet count / spleen diameter & 301 & - & 750 & 664 & \pm & 262 \\
\hline PV velocity $(\mathrm{cm} / \mathrm{sec})$ & 8 & - & 16.5 & 11.906 & \pm & 2.512 \\
\hline HA resistive index & 0.6 & - & 0.9 & 0.739 & \pm & 0.082 \\
\hline Spleen Diameter (mm) & 110 & - & 240 & 147.390 & \pm & 20.836 \\
\hline
\end{tabular}

Hb: Hemoglobin.WBC: White blood cells.INR: international normalized ratio. PV: Portal vein. HA: Hepatic artery.

Table.2 Endoscopy grades of varices of studied patients

\begin{tabular}{|r|c|c||}
\hline \multicolumn{3}{|c|}{ Endoscopy Grade } \\
\hline & $\mathbf{N}$ & $\%$ \\
\hline No & 43 & 43.00 \\
\hline Grade I & 10 & 10.00 \\
\hline Grade II & 15 & 15.00 \\
\hline Grade III & 32 & 32.00 \\
\hline Total & 100 & 100.00 \\
\hline
\end{tabular}

\section{N: Number}


Table.3 Right lobe diameter of the liver / serum albumin of studied patients

\begin{tabular}{|c|c|c|c|c|c|c|c|c|c|c|c|c|c|}
\hline & \multicolumn{11}{|c|}{ Endoscopy Grade } & \multirow{2}{*}{\multicolumn{2}{|c|}{ ANOVA }} \\
\hline & \multicolumn{3}{|c|}{ No } & \multicolumn{3}{|c|}{ Grade I } & \multicolumn{2}{|c|}{ Grade II } & \multicolumn{3}{|c|}{ Grade III } & & \\
\hline & Mean & \pm & SD & Mean & \pm & SD & Mean & \pm SD & Mean & \pm & SD & $\mathbf{F}$ & P-value \\
\hline $\begin{array}{l}\text { Rt lobe / } \\
\text { Albumin }\end{array}$ & 4.116 & \pm & 0.502 & 4.291 & \pm & 0.842 & 4.871 & $\pm \quad 0.971$ & 5.073 & \pm & 0.742 & 12.920 & $<0.001 *$ \\
\hline \multicolumn{14}{|c|}{ TUKEY'S Test } \\
\hline \multicolumn{2}{|c|}{ No \&I } & & \multicolumn{2}{|c|}{ No \&II } & \multicolumn{2}{|c|}{ No \&III } & & I\&II & \multicolumn{3}{|c|}{ I\&III } & \multicolumn{2}{|c|}{ II\&III } \\
\hline \multicolumn{2}{|c|}{0.893} & & \multicolumn{2}{|c|}{$0.003 *$} & \multicolumn{2}{|c|}{$<0.001 *$} & & 0.186 & \multicolumn{3}{|c|}{$0.014^{*}$} & \multicolumn{2}{|c|}{0.793} \\
\hline
\end{tabular}

SD: Standard deviation. No: Novarices.

Table.4 Platelet count/Spleen diameter ratio (PC/SD) of studied patients

\begin{tabular}{|c|c|c|c|c|c|c|c|c|c|c|c|c|}
\hline & \multicolumn{10}{|c|}{ Endoscopy Grade } & \multirow{2}{*}{\multicolumn{2}{|c|}{ ANOVA }} \\
\hline & \multicolumn{3}{|c|}{ No } & \multicolumn{2}{|c|}{ Grade I } & \multicolumn{2}{|c|}{ Grade II } & \multicolumn{3}{|c|}{ Grade III } & & \\
\hline & Mean & \pm & SD & Mean & \pm SD & Mean & \pm SD & Mean & \pm & SD & $\mathbf{F}$ & P-value \\
\hline Platelet / spleen diameter & 866 & \pm & 197 & 850 & \pm 173 & 473 & $\pm \quad 56$ & 425 & \pm & 110 & 61.774 & $<0.001^{*}$ \\
\hline \multicolumn{13}{|c|}{ TUKEY'S Test } \\
\hline \multicolumn{2}{|l|}{ No \&I } & \multicolumn{3}{|c|}{ No \&II } & \multicolumn{2}{|c|}{ No \&III } & I\&II & \multicolumn{3}{|c|}{ I\&III } & \multicolumn{2}{|c|}{ II\&III } \\
\hline \multicolumn{2}{|l|}{0.992} & \multicolumn{3}{|c|}{$<0.001 *$} & $<0.001 *$ & \multicolumn{2}{|r|}{$<0.001 *$} & \multicolumn{3}{|c|}{$<0.001 *$} & \multicolumn{2}{|c|}{0.749} \\
\hline
\end{tabular}


Table.5 Portal vein velocity of studied patients

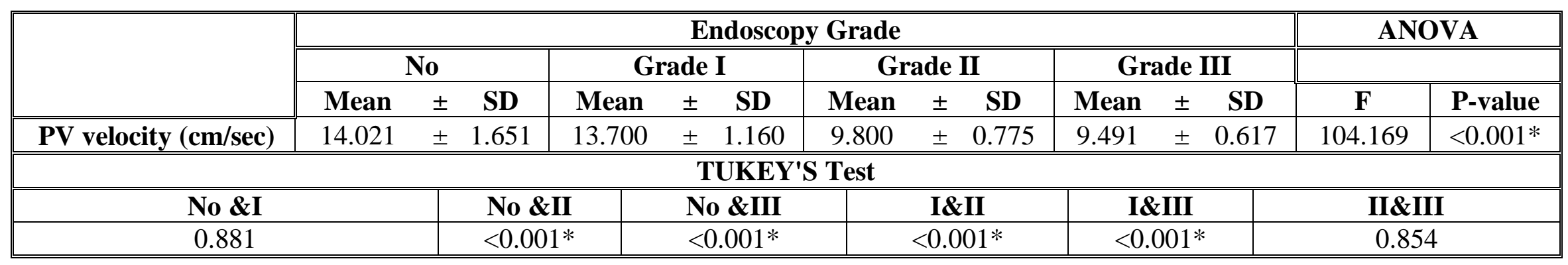

Table.6 Hepatic artery resistive index: of studied patients

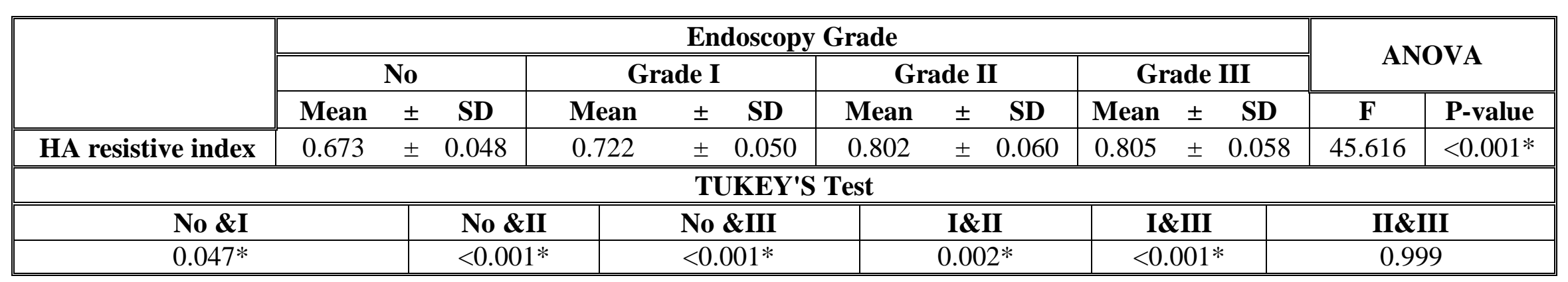

HA: Hepatic artery.

Table.7 Grades of OV with respect to Child score

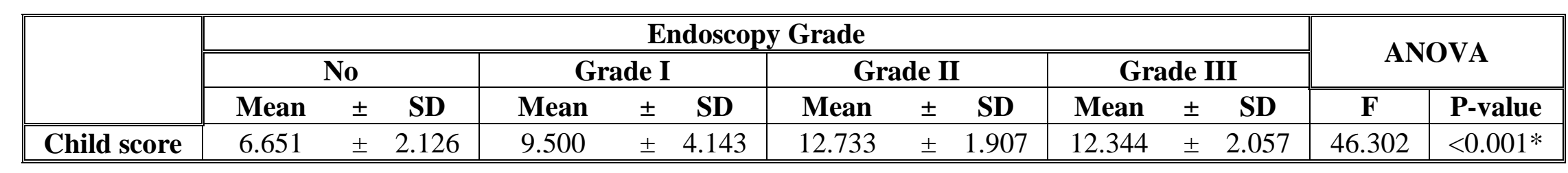


Fig.1 Receiver Operator Characteristic (ROC) curve for evaluation of right lobe diameter of the liver /serum albumin as diagnostic parameter for the presence of OV

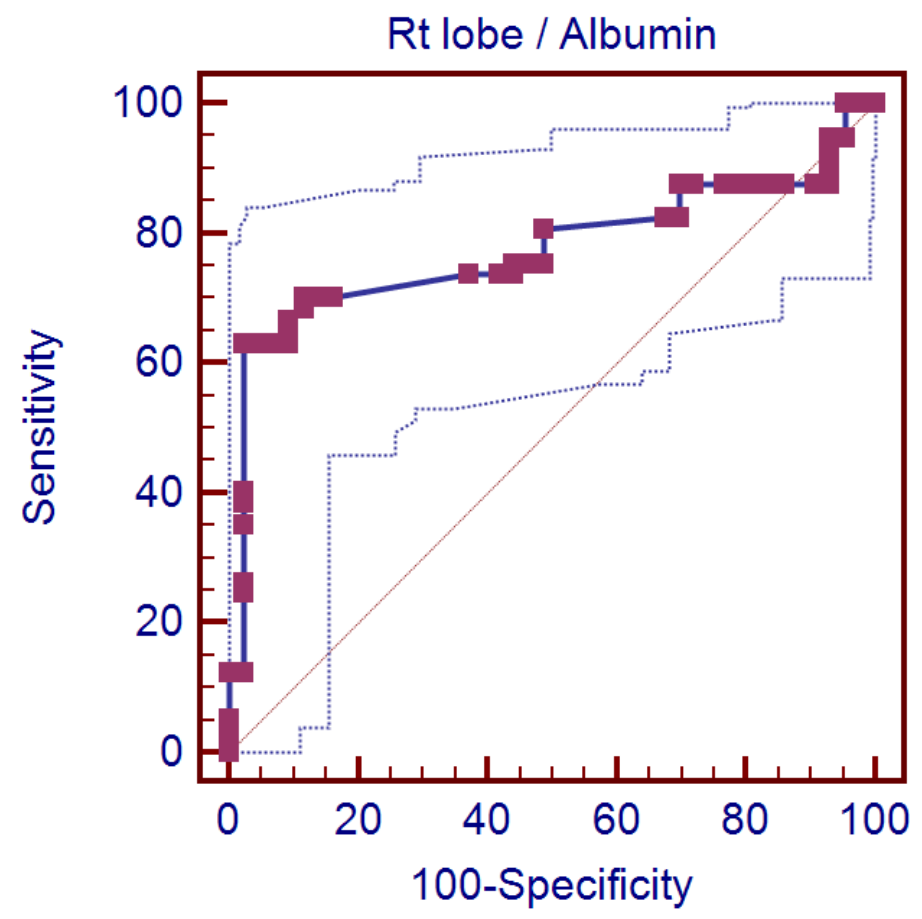

Fig.2 Receiver Operator Characteristic (ROC) curve for evaluation of Platelet count /bipolar spleen diameter as diagnostic parameter for the presence of $\mathrm{OV}$

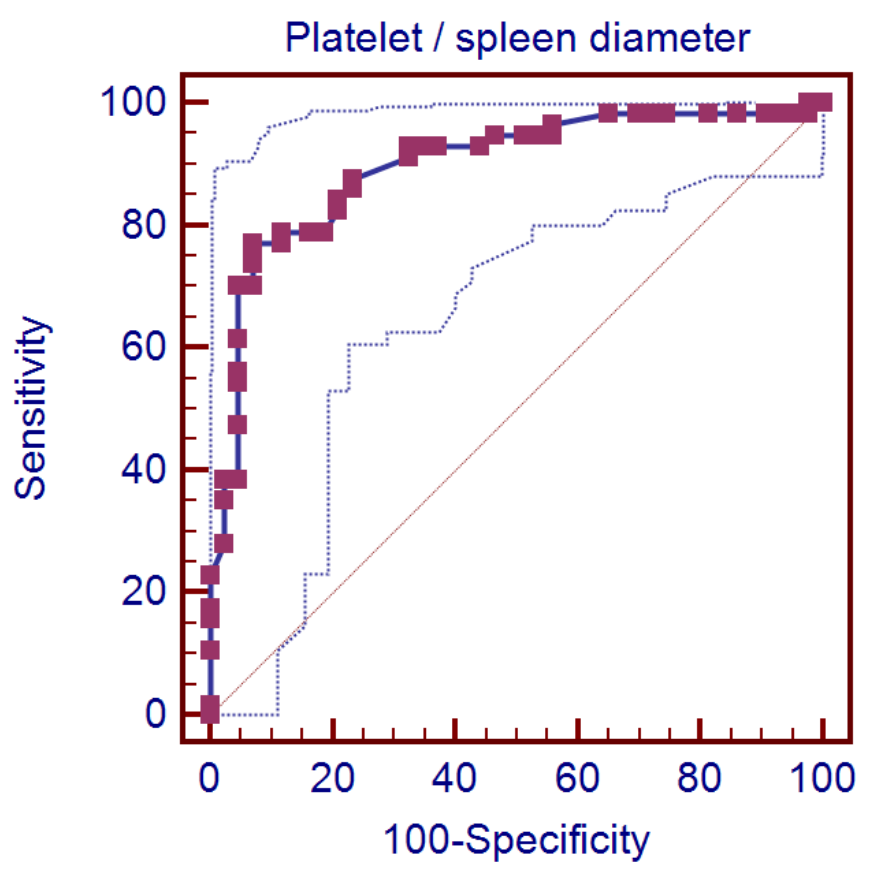


Fig.3 Receiver Operator Characteristic (ROC) curve for evaluation of portal vein velocity as diagnostic parameter for the presence of $\mathrm{OV}$

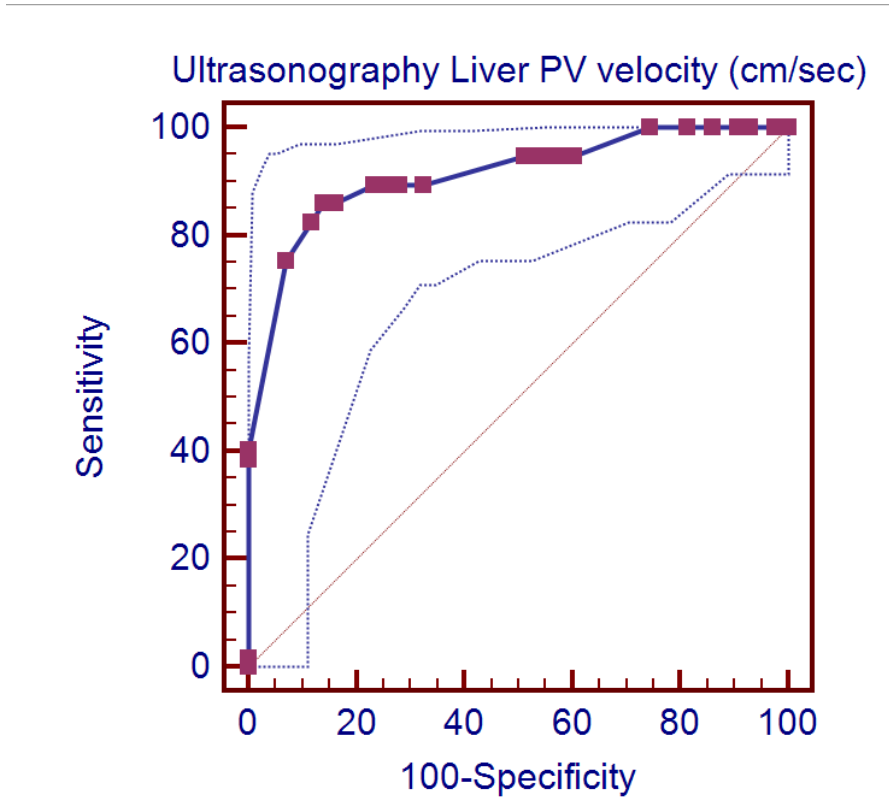

Fig.4 Receiver Operator Characteristic (ROC) curve for evaluation of hepatic artery resistive index as diagnostic parameter for the presence of $\mathrm{OV}$

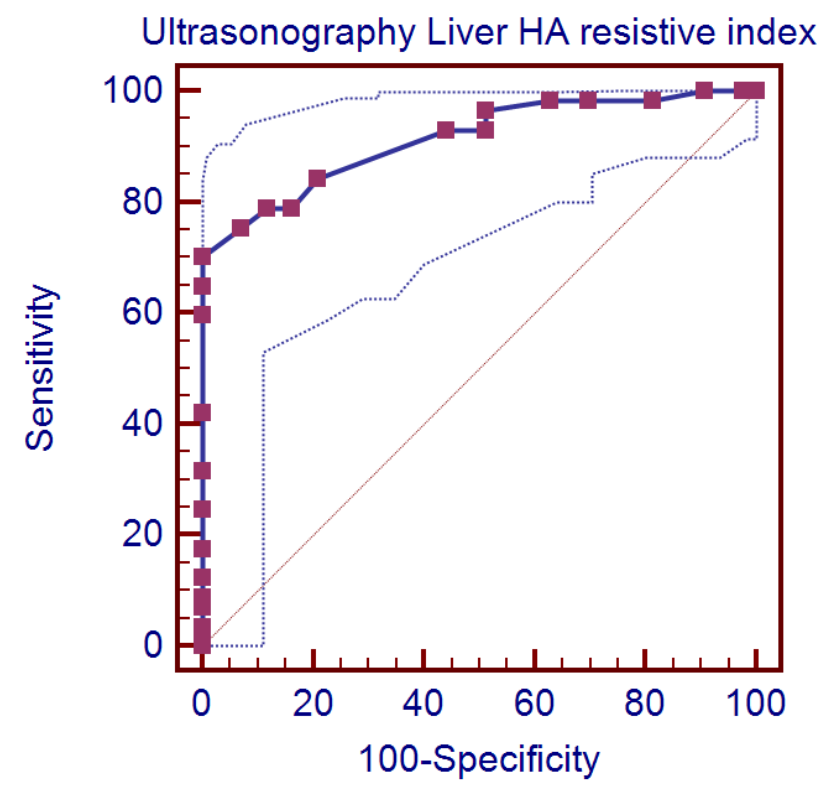


Fig.5 Correlation between right lobe diameter of liver/serum albumin \& platelet count /spleen diameter (PC/SD)

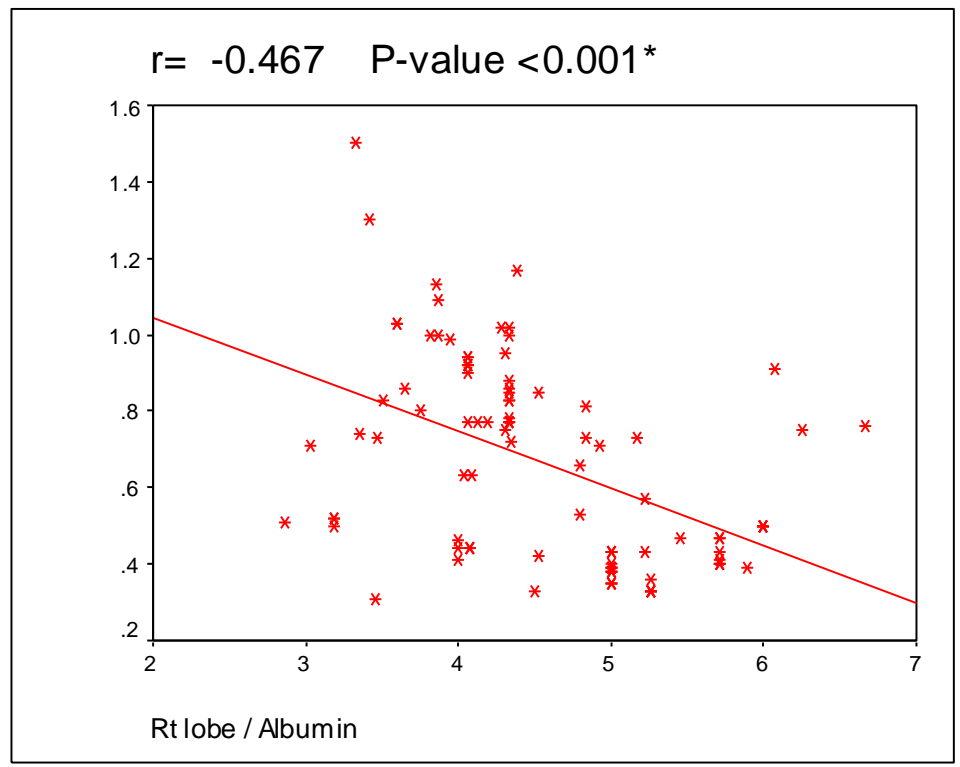

This was a comparative study aimed to evaluate right lobe diameter of the liver /serum albumin, platelet count /spleen diameter, portal vein velocity and hepatic artery resistive index as possible predictors for presence of varices in cirrhotic patients. The work was carried out on 100 cirrhotic patients.43 patients were found to have no varices, 10 patients had grade I varices, 15 patients had grade II varices and 32 patients had grade III varices.

In this study, we found a statistically significant difference between studied groups as regards portal vein velocity (PVV). A cutoff value $12 \mathrm{~cm} / \mathrm{sec}$ was found to significantly predict $\mathrm{EV}$ with $85.96 \%$ sensitivity, $86.05 \%$ specificity, PPV $89.1 \%$, and NPV $82.2 \%$. These results are in agreement with those of Berzigotti et al., (2008) who found that reduced portal vein velocity (maximal and mean velocimetry of portal vein flow, respectively $<16 \mathrm{~cm} / \mathrm{s}$ and $<10-12 \mathrm{~cm} / \mathrm{s}$ ) was helpful for diagnosis of OV. The findings of our study are in agreement with those of the study by Puneet et al, (2011), who found that mean portal vein velocity was lower in patients with $\mathrm{EV}$ as compared to those without EV.

On the other hand, the study performed by De Bem et al., (2006) revealed no correlation between portal vein velocity and the presence of gastroesophageal varices in cirrhotic patients.

Another Doppler parameter, the Hepatic artery resistive index (HARI) was performed in our study, we found statistical significance between this parameter and presence of EV with a cutoff value of 0.76 . The cut off value had $70.18 \%$ sensitivity, $100 \%$ specificity, PPV of $100 \%$ and NPV $71.7 \%$.

These results are in agreement with those of Tarzamni et al., (2008) who evaluated parameters of portal hypertension in 85 cirrhotic patients using colour Doppler ultrasonography and found that HARI is a good predictor of EV. On the other hand, Schneider et al, (1999) found no correlation between resistive index of the hepatic artery and superior mesenteric artery and HVPG, 
or between the presence and size of varices and portal vein measurements.

Another significant parameter we studied was the platelet count / spleen diameter (PC/SD).We found a statistically significant relation to the presence and grade of $\mathrm{EV}$ (p.value<0.001) at a cutoff value 570, with sensitivity $77.19 \%$, specificity $93.02 \% \mathrm{PPV}$ 93.6\%, NPV 75.5\%.These results are in agreement with those of De Franchis R and Dell'Era, (2007) who found that platelet count/spleen diameter ratio is considered the best non-invasive predictor of $\mathrm{EV}$ development.

Also Giannini et al., (2003) conducted a study in which average platelet count to spleen diameter ratio in patients who had not developed EV was 1638 and average platelet count to spleen diameter ratio in patients who had developed OV was 533.They used a cut off value of platelet count/spleen diameter ratio of 909 to predict the presence of esophageal varices with a sensitivity of $100 \%$ and $93 \%$ specificity.

Similarly, average platelet count to spleen diameter ratio in patients who had no $\mathrm{EV}$ was 2408.7 and average platelet count to spleen diameter ratio in patients who had EV was 862.6 in the study by Legasto et al., (2006).

Agha et al.,(2009) ${ }^{31}$ studied114 compensated cirrhotic patients with hepatitis $\mathrm{C}$, they reported that a cut-off value of 909 $(\mathrm{NPV}=100 \%$ and $\mathrm{PPV}=93.8 \%$ ) was effective in the diagnosis of EV. Baig et al., (2008) in their study stated that a cut-off value of 1014 can predict presence of OV.

On the other hand, results of this study are against the results of Sarwar et al., (2004) who found sensitivity and specificity of platelet count/splenic diameter to predict esophageal varices with a cut off value of 909 to be $13 \%$ and $77 \%$ respectively, which is far from significant.

Regarding the role of right lobe diameter of the liver /serum albumin as a non-invasive, inexpensive and simple predictor of $\mathrm{EV}$, we found that there was a statistically significant difference between EV grades of the studied patients as regards the right lobe diameter of the liver /serum albumin $(\mathrm{p}<0.001)$ and that there was a direct correlation between them.

We also found a cutoff value 4.92 could significantly predict OV with sensitivity $63.61 \%$, specificity $97.67 \%$ PPV $97.3 \%$, and NPV $66.7 \%$.

These results are congruent with those of Alempijevi et al., (2007) who found the grades of varices to increase as the right liver lobe/ albumin ratio increases. Considering the right liver lobe/albumin ratio cut-off value of 4.42 , this had a sensitivity of $83.3 \%$ and specificity of $29.5 \%$ for patients with varices requiring prophylactic endoscopic management.

On the other hand El Ray A. et al., 2015 found that right liver lobe diameter/ serum albumin had no role in prediction of $\mathrm{EV}$ presence.

This study introduces four easy, noninvasive methods that when used in combination can predict the presence of esophageal varices and decrease the burden of screening endoscopy for all cirrhotic patients.

Our study has some limitations. This was a single center study of a tertiary care setting, raising the question of generalizability. Also, larger studies on larger groups of patients are needed to confirm the results. 
In conclusion, Right liver lobe diameter /serum albumin, platelet count /spleen diameter and the Doppler US parameters; portal vein velocity and hepatic artery resistive index should be performed for all cirrhotic patients as they are easy to perform, inexpensive and can predict the presence of esophageal varices.

Ethical Approval: The study protocol was approved by the ethical committee of faculty of medicine, Tanta University. Informed consent was obtained from each patient before participation in the study.

\section{References}

Tsochatzis, E.A., Bosch, J., Burroughs, A.K. 2014. Liver cirrhosis. Lancet, 383: 1749-1761.

Arguedas, M.R., Heudebert, G.R., Eloubeidi, M.A. 2002. Costeffectiveness of screening, surveillance, and primary prophylaxis strategies for esophageal varices. Am. J. Gastroenterol., 97: 2441-2452.

Jensen, D.M. 2002. Endoscopic screening for varices in cirrhosis: findings, implications, and outcomes. Gastroenterol., 122: 1620-30.

Garcia-Tsao, G., Sanyal, A.J., Grace, N.D., et al. 2007. Prevention and management of gastroesophagealvarices and variceal hemorrhage in cirrhosis. Hepatol., 46(3): 922-38.

Navens, F., Bustami, R., Scheys, I., et al. 1998. Variceal pressure is a prospective cohort study in cirrhotic patients". Hepatol., 27: 15.

Madhotra, R., Mulcahy, H.E., Willner, I., et al. Prediction of esophageal varices in patients with cirrhosis. J. Clin. Gastroenterol., 34: 81-5.
Grgurević, I., Jukić, I., Sokol, S., et al. low specificity of platelet to spleen ratio for noninvasive prediction and characterization of esophageal varices in patients with alcoholic liver cirrhosis. Acta Med Croatic, 68(4-5):353-60.

De Franchis, R. 2008. Non-invasive (and minimally invasive) diagnosis of oesophagealvarices. J. Hepatol., 49:520-527.

Kim, S.H., Kim, Y.J., Lee, J.M., et al. 2007. Esophageal varices in patients with cirrhosis: multidetector CT esophagography-comparison with endoscopy. Radiol., 242:759-768.

Eichner, E.R. 1979. Splenic function: normal, too much and too little. Am. J. Med., 66: 311-20.

Barrera, F., Riquelme, A., Soza, A., et al. 2009. Platelet count/spleen diameter ratio for non-invasive prediction of high risk oesophageal varices in cirrhotic patients. Ann. Hepatol., 8: 325-30.

Taylor, K.J., Carpenter, D.A. 1985. The anatomy and pathology of portahepatis demonstrated by gray scale ultrasonography. Radiol., 3: 117.

Kirkwood, B.R., Sternc, J.A. Essential Medical Statistics. (2nd ed.). Malden, MA: Blackwell Publishing, pp. 288.

Kim, M.Y., Jeong, W.K., Bajk, S.K. 2014. Invasive and non-invasive diagnosis of cirrhosis and portal hypertension. World J. Gastroenterol., 20: 430015.

Ripoll, C., Casu, S., Hernández-Gea, V., et al. 2013. Variceal and other portal hypertension related bleeding. Best Pract. Res. Clin. Gastroenterol., 27: 649-64.

AkhavanRezayat, K., Mansour Ghanaei, F., Alizadeh, A., et al. 2014. Doppler 
surrogate endoscopy for screening esophageal varices in patients with cirrhosis. Hepat Mon., 14: 11237.

Chawla, S., Katz, A,. Attar, B.M., et al. 2012. Platelet count/spleen diameter ratio to predict the presence of esophageal varices in patients with cirrhosis: a systematic review. Eur. J. Gastroenterol. Hepatol., 24: 4316.

D'Amico, G., Pasta, L., Morabito, A., et al. 2014. Competing risks and prognostic stages of cirrhosis: a 25 year inception cohort study of 494 patients. Aliment Pharmacol Ther., 39: 1180-93.

Thabut, D., Rudler, M., Dib, N., et al. 2015. Multicenter prospective validation of the Baveno IV and Baveno II/III criteria in cirrhosis patients with variceal bleeding. Hepatol., 61: 1024-32.

Biecker, E. 2013. Portal hypertension and gastrointestinal bleeding: diagnosis, prevention and management. World J. Gastroenterol., 21: 5035.

Singal, A.K., Ahmad, M., Soloway, R.D. 2010. Duplex Doppler ultrasound examination of the portal venous system: an emerging novel technique for the estimation of portal vein pressure. Dig. Dis. Sci., 55: 1230-40.

Khalil, F., Khalil, K., Khalil, T., et al. 2010. Evaluation of clinical, Biochemical and ultrasound parameters in diagnosis of oesophageal varices. Med. J. Cairo Univ., 78: 105-109.

Berzigotti, A., Gilabert, R., Abraldes, J.G., et al. 2008. Noninvasive prediction of clinically significant portal hypertension and esophageal varices in patients with compensated liver cirrhosis. Am. J. Gastroenterol., 103: 1159-67.

Puneet, M., Ranjana, G., Gaurav, M., et al. 2011. Association between Portal
Vein Color Doppler Findings and the Severity of Disease in Cirrhotic Patients with Portal Hypertension. Iran J. Radiol., 8: 211-217.

De Bem, R.S., Lora, F.L., De Souza, R.C.A., et al. 2006. Correlation of Doppler ultrasound of the portal system with endoscopic changes caused by portal hypertension in cirrhotic patients. Arquivos de Gastroenterologia, 43: 178-83.

Tarzamni, M.K., Somi, M.H., Farhang, S., et al. 2008. Portal hemodynamics as predictors of high risk esophageal varices in cirrhotic patients. World J. Gastroenterol., 14(12): 1898-1902.

Schneider, A.W., Kalk, J.F., Klein, C.P. 1999. Hepatic arterial pulsatility index in cirrhosis: correlation with portal pressure. J. Hepatol., 30: 87681.

De Franchis, R., Dell'Era, A. 2007. Diagnosis and therapy of esophageal vascular disorders. Curr. Opin. Gastroenterol., 23: 422-7.

Giannini, E., Botta, F., Borro, P., et al. 2003. Platelet count/spleen diameter ratio: proposal and validation of a noninvasive parameter to predict the presence of oesophageal varices in patients with liver cirrhosis. Gut, 52: 1200-1205.

Legasto, G.M.A., Sevilla, J., Balay, A., et al. 2006. Platelet count/ spleen diameter ratio: A noninvasive parameter to predict the presence of esophageal varices. Phil. J. Gastroenterol., 2: 33-38.

Agha, A., Anwar, E., Bashir, K., et al. 2009. External validation of the platelet count/spleen diameter ratio for the diagnosis of esophageal varices in hepatitis C virus-related cirrhosis. Dig. Dis. Sci., 54: 654-660.

Baig, W.W., Nagaraja, M.V., Varma, M., et al. 2008. Platelet count to spleen 
diameter ratio for the diagnosis of esophageal varices: Is it feasible? Can. J. Gastroenterol., 22: 825-828.

Sarwar, S., Alam, A., Khan, A.A., et al. 2004. Platelet count/splenic size ratio: Can it predict the presence of varices in patients of Cirrhosis of liver? Proceeding ShaikhZayed Postgrad. Med. Inst., 18: 21-61.

Alempijevic, T., Bulat, V., Djuranovic, S., et al. 2007. Right liver lobe/albumin ratio: contribution to non-invasive assessment of portal hypertension. World J. Gastroenterol., 13: 5331-5. 2015. Non-invasive predictors for the presence, grade and risk of bleeding from esophageal varices in patients with post hepatitic cirrhosis. J. Egypt. Soc. Parasitol., 45: 421428.

\section{How to cite this article:}

Elshazly Abd Elhady Sheta, Mohamed Yousef, Sherief Abd-Elsalam, Rania Essam Eldeen Mohamed, Ayat Ismail, Ferial El-Kalla, Loai Mansour, Abdelrahman Kobtan, Mohamed Elhendawy and Hala M. Elsabagh. Non Invasive Diagnosis of Esophageal Varices: Can it Replace Screening Endoscopy? Int.J.Curr.Microbiol.App.Sci. 5(5): 701-715. doi: http://dx.doi.org/10.20546/ijcmas.2016.505.071 